\title{
Article
}

\section{Comprehensive NGS Panel Validation for the Identification of Actionable Alterations in Adult Solid Tumors}

\author{
Paula Martínez-Fernández ${ }^{1}$, Patricia Pose ${ }^{2} \mathbb{D}$, Raquel Dolz-Gaitón ${ }^{2} \mathbb{D}$, Arantxa García ${ }^{3}$, \\ Inmaculada Trigo-Sánchez ${ }^{4}$, Enrique Rodríguez-Zarco ${ }^{4}$ D, MJose Garcia-Ruiz ${ }^{1}$, Ibon Barba ${ }^{1}$, \\ Marta Izquierdo-García ${ }^{1}$, Jennifer Valero-Garcia ${ }^{1}$, Carlos Ruiz ${ }^{1}$, Marián Lázaro ${ }^{1}$, Paula Carbonell ${ }^{1}$ (D), \\ Pablo Gargallo ${ }^{1}$ (D) , Carlos Méndez ${ }^{5}$, Juan José Ríos-Martín ${ }^{4}$, Alberto Palmeiro-Uriach ${ }^{6}$, Natalia Camarasa-Lillo ${ }^{7}$, \\ Jerónimo Forteza-Vila ${ }^{8}$ and Inés Calabria ${ }^{1, *}$
}

Citation: Martínez-Fernández, P.; Pose, P.; Dolz-Gaitón, R.; García, A.; Trigo-Sánchez, I.; Rodríguez-Zarco, E.; Garcia-Ruiz, M.; Barba, I.; Izquierdo-García, M.; Valero-Garcia, J.; et al. Comprehensive NGS Panel Validation for the Identification of Actionable Alterations in Adult Solid Tumors. J. Pers. Med. 2021, 11, 360. https://doi.org/10.3390/jpm11050360

Academic Editor: Mirosław

J. Szczepański

Received: 24 March 2021

Accepted: 26 April 2021

Published: 29 April 2021

Publisher's Note: MDPI stays neutral with regard to jurisdictional claims in published maps and institutional affiliations.

Copyright: (c) 2021 by the authors Licensee MDPI, Basel, Switzerland This article is an open access article distributed under the terms and conditions of the Creative Commons Attribution (CC BY) license (https:// creativecommons.org/licenses/by/ $4.0 /)$.
1 Imegen-Health in Code Group, 46980 Paterna, Spain; paula.martinez@imegen.es (P.M.-F.); mariajose.garcia@imegen.es (M.G.-R.); ibon.barba@imegen.es (I.B.); marta.izquierdo@imegen.es (M.I.-G.); jennifer.valero@imegen.es (J.V.-G.); carlos.ruiz@imegen.es (C.R.); marian.lazaro@imegen.es (M.L.); paula.carbonell@imegen.es (P.C.); pablo.gargallo@imegen.es (P.G.)

2 Servicio de Anatomía Patológica, Hospital Universitario de la Ribera, 46600 Alcira, Spain; p.pose14@gmail.com (P.P.); rdolzgaiton@gmail.com (R.D.-G.)

3 Servicio de Genética Molecular y Radiobiología, Centro Oncológico de Galicia, 15009 A Coruña, Spain; arantxa.garcia@cog.es

4 Servicio de Anatomía Patológica, Hospital Universitario Virgen Macarena, 41009 Sevilla, Spain; intrigo@us.es (I.T.-S.); enriquerodriguezzarco@gmail.com (E.R.-Z.); jjrios@us.es (J.J.R.-M.)

5 Servicio de Oncología Médica, Centro Oncológico de Galicia, 15009 A Coruña, Spain; carlos.mendez@cog.es

6 Laboratorio de Anatomía Patológica, Hospital General Universitario de Castellón, 12004 Castellón, Spain; a.alpaur@gmail.com

7 Anatomía Patológica, Hospital Lluís Alcanyís de Xàtiva, 46800 Xàtiva, Spain; ncamarasalillo@gmail.com

8 Anatomía Patológica, Universidade de Santiago de Compostela, 15705 Santiago de Compostela, Spain; jeronimo.forteza@gmail.com

* Correspondence: ines.calabria@imegen.es

Abstract: The increasing identification of driver oncogenic alterations and progress of targeted therapies addresses the need of comprehensive alternatives to standard molecular methods. The translation into clinical practice of next-generation sequencing (NGS) panels is actually challenged by the compliance of high quality standards for clinical accreditation. Herein, we present the analytical and clinical feasibility study of a hybridization capture-based NGS panel (Action OncoKitDx) for the analysis of somatic mutations, copy number variants (CNVs), fusions, pharmacogenetic SNPs and Microsatellite Instability (MSI) determination in formalin-fixed paraffin-embedded (FFPE) tumor samples. A total of 64 samples were submitted to extensive analytical validation for the identification of previously known variants. An additional set of 166 tumor and patient-matched normal samples were sequenced to assess the clinical utility of the assay across different tumor types. The panel demonstrated good specificity, sensitivity, reproducibility, and repeatability for the identification of all biomarkers analyzed and the 5\% limit of detection set was validated. Among the clinical cohorts, the assay revealed pathogenic genomic alterations in $97 \%$ of patient cases, and in $82.7 \%$, at least one clinically relevant variant was detected. The validation of accuracy and robustness of this assay supports the Action OncoKitDx's utility in adult solid tumors.

Keywords: next-generation sequencing (NGS); validation; gene panels; actionable mutations; precision oncology; targeted therapies; adult solid tumors

\section{Introduction}

Cancer can rely on different genetic aberrations, including point mutations, focal or complete chromosomal copy number variants, rearrangements or structural variants. Although some of them are well understood, the growing knowledge on less frequent 
pathogenic variants constituting driver events in tumorigenesis, together with the rapid development of targeted therapies, urges clinical testing to provide broader strategies [1].

Traditional molecular diagnosis based on low-throughput techniques for the detection of single nucleotide or small deletions and insertions is now being replaced by the pressing need of personalized medicine. Given the heterogeneity and genetic complexity that many tumors present, the integration of clinical, pathological, and molecular data provides key information to outline a specific biological profile that could be crucial in clinical decision-making. The current availability and cost-effectiveness of massively parallel next-generation sequencing (NGS) and the improvement of bioinformatic data analysis programs have enabled the implementation to the clinical practice of high-throughput, sensitive, and accurate tumor profiling [2].

Here we present the analytical validation and clinical utility of the Action OncoKitDx, a hybrid capture NGS-based panel indicated in the study of adult solid tumors. The Action OncoKitDx allows, through a single analysis, a comprehensive approach of the main actionable biomarkers relevant to oncological diagnosis, prognosis, and therapy. Furthermore, it includes the detection of somatic variants that represent eligibility criteria for the inclusion in several ongoing clinical trials.

This NGS panel integrates the complete sequencing of the 50 most relevant genes in adult cancer, the analysis of gene fusions and rearrangements related to targeted therapies, the detection of copy number variants (CNVs) throughout the genome, the analysis of microsatellite instability (MSI), and the pharmacogenetics associated with toxicity or efficacy of treatments used in oncology, allowing to obtain information of great utility in clinical practice.

Here we demonstrate the sensitivity, specificity, accuracy, and reproducibility of the Action OncoKitDx for the detection of clinically actionable variants.

\section{Materials and Methods}

\subsection{Panel Design}

The panel targets all tumor suppressors or oncogenes currently included in the standard of care of clinical diagnosis laboratories for a wide range of adult tumoral types. Besides including the main actionable biomarkers relevant for the therapy, prognosis, diagnosis, and resistance monitoring of cancer, determined by the National Comprehensive Cancer Network (NCCN, https:/ / www.nccn.org/, accessed on 24 March 2021) and Spanish Society of Medical Oncology (SEOM, https:// seom.org/, accessed on 24 March 2021) guidelines or related to approved treatments by international organizations as the Food \& Drug Administration (FDA; https:/ /www.fda.gov/, accessed on 24 March 2021) and the European Medicines Agency (EMA; https: / / www.ema.europa.eu/, accessed on 24 March 2021), it also interrogates many other genes related to tumorigenesis and potential targets for therapies being tested in clinical trials or susceptible to be developed in the future.

The complete sequencing of the codifying region of a total of 50 genes identifies point mutations, including single-nucleotide variants and small indels, among $A K T 1, A K T 2$, AKT3, ALK, ARID1A, ATRX, BRAF, BRCA1, BRCA2, CDH1, CTNNB1, EGFR, ERBB2/HER2, ESR1, ESR2, FGFR1, FGFR2, FGFR3, FGFR4, GNA11, GNAQ, HIST1H3H, HRAS, IDH1, IDH2, KIT, KRAS, MAP2K1, MET, MTOR, MYC, MYCN, NRAS, NTRK1, NTRK2, NTRK3, PDGFRA, PIK3CA, PBRM1, PMS2, PTEN, POLD1, POLE, RET, ROS1, SDHA, SDHB, TERT (promoter included), TP53, and VHL. The detection of CNVs throughout the genome, from gains and losses of chromosomes or complete chromosomal arms, to specific genes and even exons, relies on three approaches: coverage of the 50 target genes, 500 SNPs distributed across the genome and off-target readings. It also includes the detection of fusion genes and breakpoint identification on selected intronic regions of $A L K, B C R, B R A F$, EGFR, NTRK1, NTRK2, ETV6, RET, and ROS1 with any other partner of the genome. The Action OncoKitDx also allows the determination of MSI by the analysis of 110 markers, and pharmacogenetics associated with toxicity or efficacy of treatments used in oncology by analyzing ten high-evidence SNPs according to PharmGKB. 


\subsection{Sample Selection}

For evaluation of the analytical performance of the assay, samples previously or subsequently analyzed with different methodologies and reference materials from Coriell Cell Repositories and Horizon Dx were also screened with the Action OncoKitDx. A total of 64 samples from different formalin-fixed paraffin-embedded (FFPE) tumor specimens, fresh frozen tissue, and peripheral blood were employed. Sensibility and specificity were assessed in 10 samples for point mutation and small indel detection, 10 samples for CNVs, 5 samples for fusions and large rearrangements, 6 samples for pharmacogenetics, and 45 samples for microsatellite instability evaluation. Both the pharmacogenetic and the $\mathrm{CNV}$ analyses used samples from previous sections. Likewise, the repeatability and reproducibility evaluation were carried out with samples previously analyzed in this validation.

To evaluate the assay's performance in clinical specimens, tumor and patient-matched control samples from some of the most frequent adult solid cancer types were screened for mutations, including breast cancer, colorectal cancer, gastrointestinal stromal tumor, lung cancer, melanoma, ovarian cancer, pancreatic cancer, and other tumor types (endometrium, cervix, esophagus, larynx, prostate, kidney, thyroid, and bladder). Specifically, 138 tumor samples and 28 controls selected from different Spanish hospitals were included in this validation.

\subsection{DNA Extraction and Quality Control}

Selected samples were submitted to a pathological review in order to estimate the tumor cellularity. Genomic DNA (gDNA) from tumor and control tissue was extracted using the commercial extraction kits RecoverAll ${ }^{\mathrm{TM}}$ Total Nucleic Acid Isolation Kit for FFPE (Thermo Fisher Scientific, Waltham, MA, USA) and the QIAamp DNA Investigator Kit (Qiagen). Concentration was measured by fluorometric quantification using Qubit Fluorimeter (Thermo Fisher Scientific) with Qubit dsDNA BR Assay kit and Qubit dsDNA HS Assay kit (Invitrogen). DNA Integrity Number (DIN) was determined using the DNA ScreenTape assay (Agilent Technologies). Despite the recommended cut-off DIN value of 3 by the Action OncoKitDx user guide, samples with a DIN value above 2 were considered.

\subsection{Library Preparation and Next-Generation Sequencing}

The custom panel uses the SureSelect XT HS kit (Agilent), a commercial kit specially designed for small amounts of FFPE DNA as input and detect low allelic frequencies. After the mechanic fragmentation of 50-200 ng of tumoral gDNA from each sample to an average of 200 bases using the ME220 Focused-ultrasonicatorTM (Covaris), library preparation followed the Action OncoKitDx user guide. For the obtention of indexed libraries by molecular barcoding, DNA fragment ends were repaired and adenylated. Ligation of adapters incorporated unique molecular indexes (UMIs), specific DNA sequences that allow discrimination of each dsDNA molecule. Agencourt AMPure XP beads (Beckman Coulter) that selectively bind DNA $100 \mathrm{bp}$ and larger were used for purification. Libraries were universally amplified by PCR (10-16 cycles, depending on the quality and amount of input DNA) with adapter-specific primers for the union of indexes, universal indexes, and sequencing adapters. Indexes are 8-nucleotide unique sequences compatible with Illumina adapters. They mark each sample's library providing a unique combination, together with the universal indexes, that will allow bioinformatic analysis after sequencing. Indexed DNA fragments were then purified. Library quality control was performed on Tapestation 2000 and the commercial kits D1000 reagents and D1000 ScreenTape (Agilent). A mix of biotinylated capture probes specifically designed for the Action OncoKitDx were hybridized to regions of interest and then captured with streptavidin beads. Library enrichment involved the post-capture PCR amplification (12 cycles) and purification of products with Agencourt AMPure XP beads (Beckman Coulter). Libraries were validated and quantified using Qubit 2.0 fluorometer, Qubit ds DNA HS Assay kit (Invitrogen). A denaturation protocol was carried out prior to sequencing, using the NextSeq 500/550 Mid Output v2.5 and NextSeq 500/550 High Output v2.5 sequencing kits (Illumina) and Phix 
control (Illumina), and libraries were diluted to $1.5 \mathrm{pM}$. Pools were then loaded into the NextSeq 550 system (Illumina) for massive library sequencing in "Stand-alone" mode with $2 \times 75$ paired-end reads following the manufacturer's instructions.

\subsection{Bioinformatic Pipeline}

The Sample Sheet necessary for sequencing was generated using the Illumina Experiment Manager (IEM) software version 1.14.0 (Illumina). Molecular barcoding allowed the elimination of optical (sequencing) and PCR duplicates without removing duplicates from different DNA molecules. The monitorization of sequencing run quality was based on the Q30 value and cluster pass filter, expecting measures greater than $80 \%$ and $70 \%$ respectively. The FASTQ files generated followed a quality evaluation applying the FastQC v0.11.5 software (Babraham Bioinformatics), measuring parameters such as the average quality per position of all readings, percentage of duplicate reads, sequence repeats patterns, etc. QC metrics were also evaluated from the BAM file of each sample in terms of uniformity, average coverage, and percentage of the region covered at $100 \times$ Bioinformatic analysis, including the alignment to the reference sequence Genome Reference Consortium Human Build 37 (GRCh37), annotation and variant calling, followed a self-developed pipeline through the DataGenomics platform.

For the CNV analysis, in-house scripts were used to obtain a fractional coverage based on a correlation between the number of normalized readings of a region with respect to the number of DNA copies for that region. A minimum inter-sample variability was guaranteed by homogenizing experimental conditions between different samples and genomic regions. CNV calls were classified by DataGenomics based on their credibility using a scoring algorithm that takes into account parameters as $\log _{2}$ ratio, event size, proximity, and type of contiguous events. Copy number was calculated upon the sample's tumor cellularity. $\mathrm{CNV}$ plots provided by the platform were manually reviewed to discard possible artifacts and validated by digital PCR or MLPA. The Action OncoKitDx allowed the analysis of CNVs regarding genes included in the panel as well as the study of loss of heterozygosity with a neutral copy number (Copy-neutral LOH) by means of a SNP array evenly distributed throughout the genome.

For structural variant analysis, probes were designed on intronic regions with high probability of presenting fusions. This methodology enabled the identification of any rearrangement in all covered regions. The detection was performed using the BAM alignment file and LUMPY software version 0.2.13, identifying two types of readings surrounding structural events: split reads and discordant read pairs. Distance between discordant read pairs and presence of split reads was used to detect the type of structural event and fusion breakpoint. In combination, they both allowed fusion identification, in addition to other types of large rearrangements, such as deletions or duplications. Artifacts were discarded by quality score assigned by DataGenomics based on split and mate split reads coverage.

For MSI analysis, a panel was designed including 110 microsatellite markers located thorough the genome. A reference was created by determining the allelic combination of the selected regions in a set of microsatellite stable (MSS) samples. Each microsatellite region was thus evaluated by comparison with the model created to categorize it into stable or unstable biomarker. A coverage threshold determined valid results, setting a quality criterion of a minimum of 99 valid markers to analyze a sample's MSI. The instability fraction is the proportion of unstable markers among the total of valid markers. Fractions between 0 and 0.17 are related to stable samples, from 0.21 to 0.30 correspond to low instability, and above 0.31 are associated to high instability. Fractions between 0.18 and 0.20 give place to inconclusive results.

\subsection{Variant Functional and Clinical Classification}

Mutation analysis was carried out using DataGenomics. This platform enables curation by automatic variant calling and annotation, and allows their prioritization based on 
quality criteria and clinical relevance. Standard filters were applied to deprioritize variants in order to ensure high-confidence calls based on: (i) technical quality (e.g., coverage $\leq 20$, allele frequency in tumor sample $\leq 0.05$ ); (ii) evidence in the internal database (i.e., artifact or benign categorization); (iii) benignity likelihood based on: population frequency $\geq 0.02$ in the GnomAD database, and protein effect (e.g., exonic synonymous or intronic non-splicing variants). All remaining variants were submitted for manual review and validation with the Integrative Genomics Viewer (IGV; The Broad Institute, Cambridge, MA, USA).

After variant assessment, functional classification of variants as either pathogenic, likely pathogenic, "variant of unknown significance", likely benign or benign was performed following the ACMG (American College of Medical Genetics and Genomics) guidelines. Supporting pathogenicity evidence included COSMIC, ClinVar, Varsome, clinical guidelines (NCCN, ESMO), clinical trials, and scientific literature review. Clinical relevance of likely pathogenic and pathogenic variants was automatically reported by Data Genomics, determined by means of the four-tiered ACMG/AMP (Association for Molecular Pathology) guideline system recommended by the Commission of Personalized Medicine (ComPerMed). Variants were classified for their implications in diagnosis (D), prognosis $(\mathrm{P})$, resistance to treatment $(\mathrm{R})$, or therapy $(\mathrm{T})$, indicating the robustness of the available evidence depending on whether it is recommended by clinical guidelines such as SEOM, NCCN (tier I) or if it is widely supported by the literature and databases (tier II).

\section{Results}

\subsection{Sequencing Performance}

The mean Q30 value of the sequencing runs was $90.7 \%$, and $88.4 \%$ the average percentage of clusters passing filter, always within the density range recommended by the manufacturer of the sequencing equipment used. The assay achieved an average coverage of $296 \times$ after analysis of UMIs. A correct uniformity was obtained with $97.43 \%$ of the bases covered at $>20 \%$ of the average coverage and $71.01 \%$ were covered at a depth of $100 \times$.

\subsection{Analytical Sensitivity and Specificity}

We validated the Action OncoKitDx's accuracy by analytical sensitivity and specificity determination. SNVs and indels were assessed through seven clinical samples. Mutations were confirmed on an amplicon-based mini panel built on the design of other already validated and marketable panels such as the Colorectal OncoKitDx, TP53 OncoKitDx, and BRCA Plus OncoKitDx. Two commercial peripheral blood samples from Coriell Institute including different known variants were diluted at 1:10 with a negative control. Mutations were screened and detected by the Action OncoKitDx. Variants with an allelic fraction above the 5\% LOD and located within the panel's target regions were detected with a mean sensitivity of $100 \%$ and specificity of $99 \%$. To support the panel's accuracy, a reference material from Horizon Dx with over 300 variants at different allelic frequencies, OncoSpan gDNA, was also sequenced. All 70 mutations matching genes included in the panel were detected with similar allelic frequencies.

For the CNV analysis, as well as for the establishment of its LOD, $616 \mathrm{CNV}$ calls including genes in the Action OncoKitDx panel were evaluated among 10 samples previously analyzed with a different NGS panel (Paediatric OncoPanel Dx) or another technique (MLPA, dPCR). Score classification was validated with a 100\% concordance between the known variants and their categorization into High-Score.

Nine known structural rearrangements were screened in five samples previously analyzed by real-time PCR or Sanger sequencing. Concordance of results determined a $100 \%$ sensitivity and specificity over $99.9 \%$.

The MSI analysis relied on 45 pre-analyzed samples by standard fragment analysis, from which 30 were correctly hybridized and met the quality criteria. Two samples were excluded for not reaching a DIN value higher than 1 . A $100 \%$ sensitivity and 95\% specificity were obtained, with 27 out of 28 concordant cases. 
Among six samples, 8 out of all the pharmacogenetic positions included in the Action OncoKitDx were validated by PCR and Sanger sequencing. All 32 positions were confirmed with $100 \%$ analytical sensitivity and specificity greater than $99.9 \%$.

\subsection{Limit-of-Detection}

Despite the Action OncoKitDx's capacity of detecting minor allelic fraction mutations, a 5\% LOD was set for SNVs and structural variants, since a considerable proportion of false negatives would be obtained below this threshold. Upon analysis of previously employed samples, the 5\% LOD was validated and the CNV detection limit was determined.

A sample with two know point mutations was diluted in a negative control of similar genomic quality to obtain a 5\% allelic frequency. Among three aliquots sequenced, both variants were detected at a frequency close to the expected VAF. For CNVs, graphic representation of the $\log 2$ ratio variation in four tumor specimens with respect to their percentage of infiltration of non-tumor cells in the sample (Figure 1) allowed the establishment of a threshold ensuring correct gain and loss copy number detection. The LOD called was 3 copies for insertions and $\leq 1$ copy for deletions in samples with up to $50 \%$ infiltration with non-tumor cells. However, in samples with a DIN $<3$ and a non-tumor cell infiltration fraction greater than $50 \%$, the LOD may be decreased.

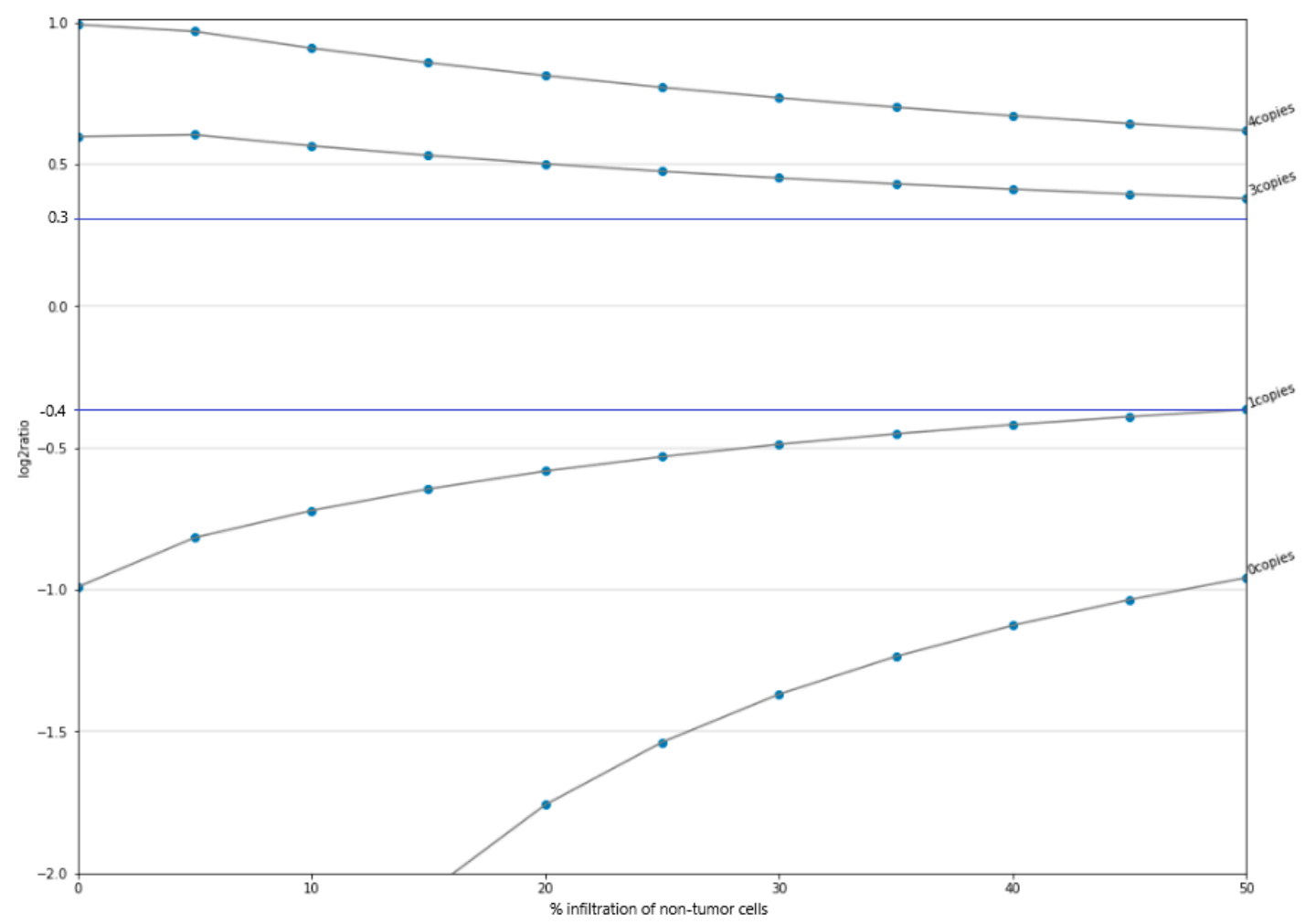

Figure 1. $\log _{2}$ ratio variation of the CNV event according to the number of copies versus percentage of infiltration of non-tumor cells in the tumor sample. Beyond 0.3 (top blue line) for insertions (i.e., number of copies $>2$ ) and below -0.4 (bottom blue line) for deletions (i.e., number of copies < 2) CNVs are detected.

\subsection{Repeatability and Reproducibility}

To assess repeatability (intra-run), one diluted sample was sequenced by triplicate in one run. Beyond evaluating that a known mutation at a $5 \%$ VAF was detected in all three aliquots with a variation coefficient lower than $25 \%$, concordance of results was observed for all variants detected. Among 118 point mutations, 117 were reported in all three, giving a concordance of $99.15 \%$. One discordant variant was excluded from the analysis for being likely an artifact due to its proximity to an homopolymer. Excluding from the analysis CNVs of size less than $0.5 \mathrm{~Kb}$ or SCORE lower than or equal to 8 , and those present on 
sex chromosomes $\mathrm{X}$ and $\mathrm{Y}$ for possible imbalance, results were concordant in all three replicates analyzed. For large rearrangements, poor quality events and those with coverage lower than 10 were excluded from the analysis. In all three samples, one deletion was detected, whose coverage and mate and split readings were consistent in the three samples. MSI was determined, resulting in a MSS fraction $100 \%$ concordant among the samples. All the pharmacogenetic positions were studied and correctly detected in the three samples.

Reproducibility (inter-run) was evaluated by analysis of three samples in two different sequencing runs, changing technicians and reagent batches as far as possible. All point mutations included in the regions covered by the Action OncoKitDx with a frequency greater than $5 \%$ and filtered by quality PASS and $\mathrm{d} 100$ by means of the Data Genomics Platform were considered. After excluding five variants due to their proximity to homopolymers, global concordance of the reproducibility results was $98.3 \%$. Low and medium quality $\mathrm{CNVs}$ were excluded, as well as events of size lower than $0.5 \mathrm{~Kb}$. Only one sample had a high-quality event, which was found in both duplicates. Three fusions were detected, each one in a different sample and were coherent in both duplicates. The three selected samples were stable for microsatellites and results were reproducible in all of them. All the pharmacogenetic positions were consistent in both tests for each of the three samples. Finally, results showed a $99.15 \%$ repeatability and $98.3 \%$ reproducibility for SNV detection, while both values were $>99.9 \%$ for CNVs, structural variants, MSI and pharmacogenetics. Both reached good robustness, with overall values greater than $99 \%$.

\subsection{Number of Samples per Run}

To calculate the number of samples that can be loaded in a run based on the guarantee of $96.2 \%$ of the bases covered at a depth of $100 \times$, a total of 26 samples were analyzed, covering a range of genomic integrities, varying from 1.3 to 8.7 .

As seen in Figure 2, beyond 26 million readings, most samples reached a plateau phase, in which the increase in the number of readings was not reflected in a significant increase in the percentage of bases covered at $100 \times$. The samples that did not reach the plateau were samples of low or medium integrity, from which starting DNA was less than recommended. Since the increase in the number of readings in these samples was not worth the reduction in the number of samples per run, 26 million readings (or 13 million clusters) were established as the minimum number of readings to carry out the Action OncoKitDx sequencing.

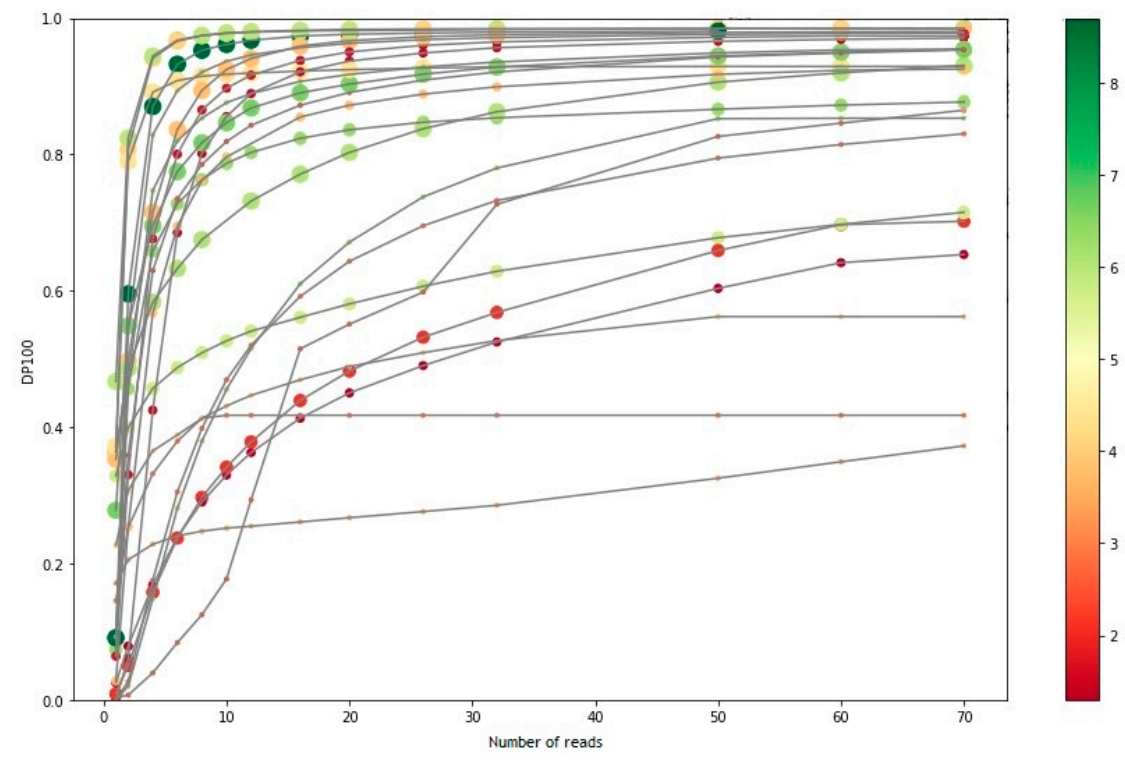

Figure 2. Graphical representation of the DP100 (fraction of bases covered at $>100 \times$ ) of different samples versus their respective number of readings. 
Thus, the maximum number of samples recommended per run to guarantee a minimum number of PF clusters of approximately 13 million per sample, which depends on the sequencing kit used, was 13 for NextSeq 500/550 Mid Output v2.5 kit and 32 for NextSeq 500/550 High Output v2.5 kit.

\subsection{Clinical Feasibility}

The validation of the assay's performance and clinical usefulness in patient samples was performed by comparison with the mutational prevalences from the Cancer Genome Atlas (TCGA) database and by assessment of the clinical potential of the results provided. Total of 14 specimens did not meet the sample or sequencing quality criteria and were excluded from the analysis. A total of 126 tumor samples and 26 controls from 104 patients presenting some of the most frequent cancer types with actionable mutations were included in the different cohorts: breast cancer $(n=14)$, colorectal cancer $(n=15)$, gastrointestinal stromal tumor $(n=4)$, lung cancer $(n=19)$, melanoma $(n=19)$, ovarian cancer $(n=10)$, pancreatic cancer $(n=12)$, and other tumor types (endometrium, cervix, esophagus, larynx, prostate, kidney, thyroid, and bladder, $n=11$ ).

The findings from the TCGA project were compared to the mutational landscape obtained within the clinical feasibility cohorts of the main tumor types or with the largest number of specimens. Our results demonstrated good overall correlation, thus allowing cautious extrapolation of the mutational prevalences obtained with the Action OncoKitDx (Figure 3).

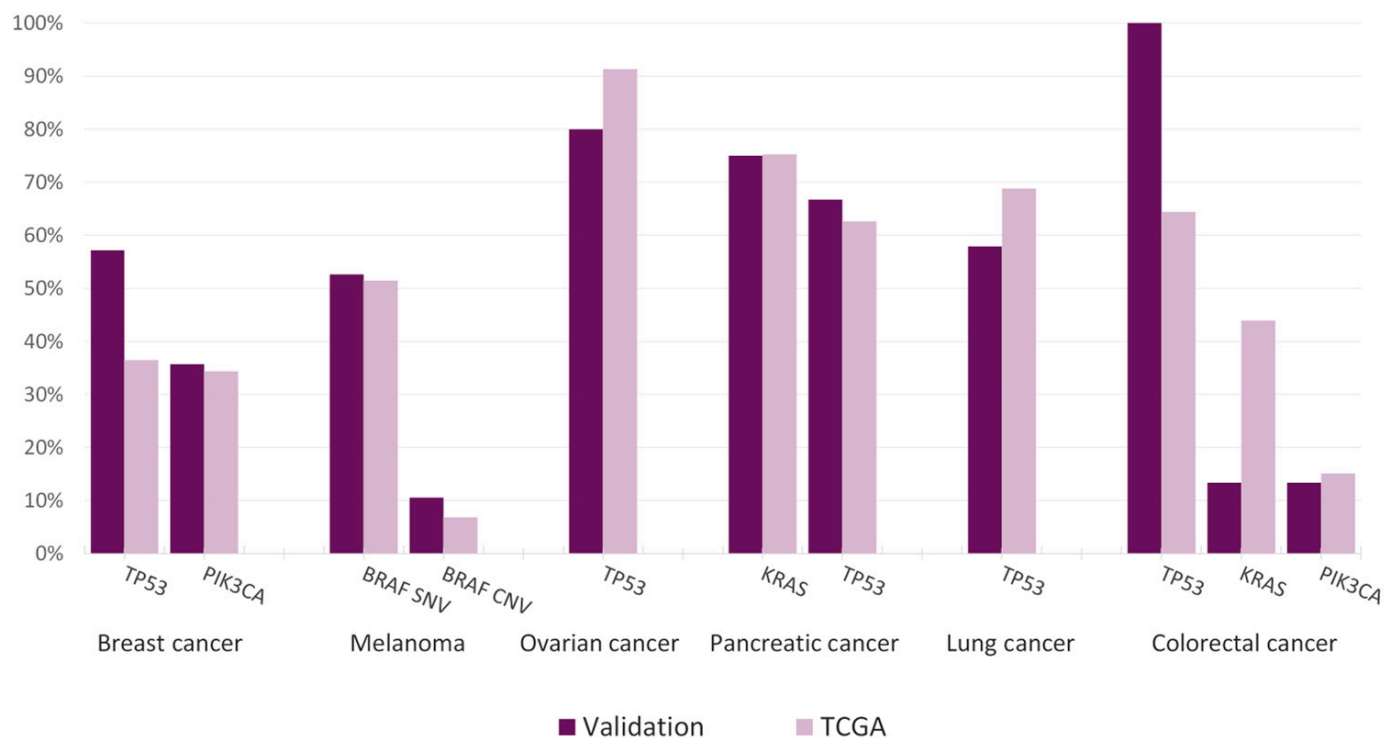

Figure 3. Comparison of the frequencies of the most frequent alterations among different tumor types between the TCGA dataset and the Action OncoKitDx clinical feasibility cohorts (breast cancer, melanoma, ovarian cancer, pancreatic cancer, lung cancer, and colorectal cancer).

In breast cancer, the two main altered genes are TP53 (36.47\%) and PIK3CA (34.35\%). Results were consistent in our cohort, where frequencies were $57.14 \%$ and $35.71 \%$ respectively. With respect to melanoma, $B R A F$ alteration was the main driver, with comparable SNV and CNV frequencies $(52.63 \%$ and $10.53 \%$ in our cohort, $51.40 \%$ and $6.80 \%$ in TCGA respectively). In ovarian cancer, TP53 alterations are almost ubiquitous (91.30\% in TCGA), being even more remarkable depending on the subtype. Here we found these aberrations in $80.00 \%$ of cases. Two of the main affected genes in pancreatic cancer according to TCGA, KRAS $(75.27 \%)$ and TP53 (62.64\%), were also found in similar proportions in our validation cohort, being $75.00 \%$ and $66.67 \%$ respectively. In lung cancer the most frequently altered gene is TP53, with a global prevalence of $68.77 \%$ across the different subtypes. Our clinical feasibility study found a similar frequency of $57.89 \%$ in the lung cancer cohort. Finally, the 
most affected genes in the colorectal cancer TCGA dataset were also observed in our cohort, being TP53 (64.37\%), KRAS (43.87\%), and PIK3CA (15.09\%). Despite observing PIK3CA at a coherent proportion (13.33\%), less consistent results were found for TP53 (100\%) and KRAS $(13.33 \%)$ where frequencies differed from the TCGA dataset.

Pathogenic or likely pathogenic variants were detected in 101 out of 104 patient cases (Figure 4). Among them, actionable mutations with known therapeutic, diagnostic, prognostic, or resistance relevance (TI, TII, DI, DII, PI, PII, RI, RII) were reported for 86 cases.

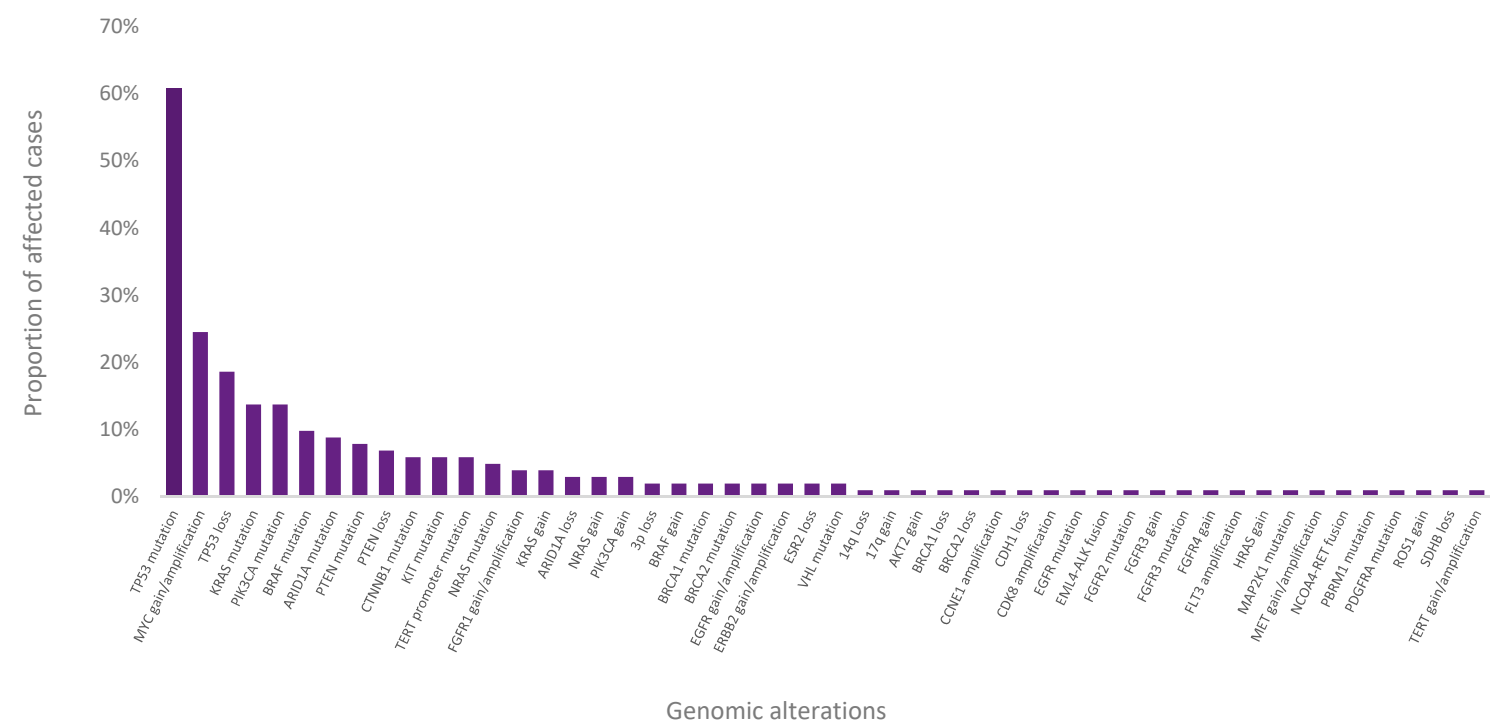

Figure 4. Frequency of the genomic alterations detected.

The PIK3CA hotspots (H1047R and E545K) were detected in 5 out of 14 samples from the breast cancer cohort, where beyond being described as an independent negative prognostic factor related to increased tumor aggressiveness, it is suggested to condition resistance to fulvestrant and identifies candidates for alpelisib plus fulvestrant therapy in HR+ and HER2- breast cancers (TI, PII, RII) [3-8]. Other investigational biomarkers include different inactivating TP53 mutations, whose subtype-dependent prognostic value is supported by different studies [9,10].

In colorectal tumors, the G12 KRAS mutation detected in 2 out of 15 samples, is predictive for a lack of response to the EGFR inhibitors cetuximab and panitumumab, associated to primary resistance (RI). Despite results on the prognostic value of this biomarker being controversial, studies suggest that KRAS activation could condition poor prognosis [11,12]. Nevertheless, its prognostic value seems to depend on the tumor location as it has been observed to indicate a poor prognosis in left-sided patients, whereas right-sided patients did not experience the same effect.

With respect to melanoma, $B R A F$ alteration is the main driver although there is a broad range of pathogenic mutations described. However, routine genetic testing is usually performed on exons 11 and 15, where actionable mutations are found. $B R A F$ mutations are associated to different recommendations on decision-making according to their location, some related to responsiveness to BRAF and MEK inhibitors (V600E/K) while others have not demonstrated response, as mutations in codons other than V600 in exons 11 or 15 . Here we found sensitizing mutations in 9 out of 19 patients (V600E/K) (TI). A T599dup variant was detected, previously identified in cases of thyroid carcinoma, metastatic melanoma, and non-small cell lung cancer but results show contradictory evidence on its sensitivity to inhibitors $[13,14]$. Moreover, other important alterations are G13 and Q61 in NRAS (TII, PI) identified in 3 patients and L576P in KIT (TI) in 1 patient. Whereas exon 11 and exon 13 mutations are recognized as sensitizing mutations to KIT inhibition, exon 17 mutations are suggested to have minimal or no sensitivity. 
The diversity of KIT alterations, including amplifications, differing in their sensibility to targeted therapy (e.g., imatinib, sunitinib, nilotinib), make comprehensive genomic profiling essential [15-18]. NRAS mutations are a prognostic factor and its therapeutic impact is under investigation. MEK inhibitors such as bimetinib appear to be a promising treatment option for NRAS-mutated melanomas [19-22].

$B R C A 2$ (TI), whose biallelic loss is associated to responsiveness to PARP inhibitors and diagnostic relevance with evidence level I, as well as prognostic implications, was altered in 2 out of 10 ovarian cancer patients. Some PIK3CA mutations detected (M1043V and H1047R) in 2 samples have approved treatment in other tumoral types, displaying the possibility of potential future therapeutic options.

KRAS G12 mutations (DII, PII) were also detected in 10 out of 12 patients of the pancreatic cancer cohort, in which it has diagnostic implications as it can be used to differentiate it from chronic pancreatitis. The presence of mutations in KRAS is also associated with lower survival and is more frequent in metastasis than in local disease, for which it is also considered of prognostic relevance [23].

Finally, the most frequently altered gene in lung cancer is TP53 (PII), unfrequently tested except in case of Li-Fraumeni syndrome suspicion and associated to prognostic relevance [24-26]. Missense, nonsense, and splicing mutations were found in 11 out of 19 patients. The analysis also allowed the detection of different characteristic alterations such as the largely described EGFR exon 19 deletion (E746_A750del) (TI) which confers sensitivity to EGFR inhibitors carried by 1 patient in this cohort. An infrequent exon $11 B R A F$ mutation (G466V) was detected in 1 patient. Despite activating $B R A F$ mutations being very prevalent in lung cancer, the impact on response to $B R A F$ inhibitors of changes other than V600 are not well documented and research is necessary.

The assay also enabled the detection of a EML4-ALK fusion (TI, RI) in a lung cancer patient. This characteristic alteration confers sensitivity to ALK inhibitors, where alectinib has demonstrated better efficacy than crizotinib, and resistance to EGFR TKIs. A less frequent fusion in colorectal cancer was also revealed. Whereas the NCOA4-RET fusion oncogene (TII, PII) found in 1 colorectal cancer patient has been described in this tumor type, it is not usually tested. This alteration could condition a worse prognosis and grant access to RET TKIs in clinical trials [27].

Some relevant copy number variants have been reported in the different cohorts. In breast cancer, FGFR1 amplification represents the most frequent genomic aberration, mainly observed in the HR+/HER2- subtype [28-30]. FGFR1 amplification is correlated with lower overall survival in HR+ breast cancer [28]. Likewise, an association between FGFR1 amplification and resistance to endocrine therapy has been described, demonstrating a worse long-term metastasis-free survival in tumors that overexpress FGFR1. More recently, FGFR1 amplification has been suggested as a predictive marker for lack of efficacy of CDK4 and CDK6 inhibitors. This alteration was not only found in 1 breast cancer patient, but also in 3 lung cancers, where despite being a frequent event particularly present in squamous cell carcinoma, no clinical value has been associated. Other amplifications with potential clinical implications in investigation were reported, as $M Y C$ in 9 melanomas, where it is an important negative prognostic factor, and EGFR in 1 breast cancer patient, present in up to $78 \%$ of triple negative cases associated with a worse prognosis and more aggressive metastases [31,32]. VHL inactivation, either by mutation or 3p deletion, was reported in 2 renal cell carcinomas. These tumors may be sensitive to VEGF receptor-targeted inhibitors and in cases where this gene is the only driver, the occurrence of metastasis is infrequent, while multiple drivers are associated with a higher probability of metastasis [33].

Off-target copy number variants were also identified. CCNE1 amplification is frequently present in high-grade serous ovarian cancer where it has a certain diagnostic value as it is generally mutually excluding with $B R C A$ inactivation. This alteration was found in 1 patient of the ovarian cancer cohort $[34,35]$.

Two MSI-H tumors were identified in the colorectal cancer cohort, where it is a major carcinogenetic pathway, and two MSI-L tumors were found among the renal cell and 
ovarian cancer groups. The MSI-H reflects a tumor mutator phenotype owed to Mismatch Repair deficiencies (dMMR) [36]. Despite MSI-H being present in most solid tumor types, its prevalence is highly variable. This phenotype, observed in glioblastoma, NSCLC, esophageal, breast, and ovarian cancer is relatively common in CRC, endometrial, and gastric cancer [37,38].

Among the three methods available to determine the MSI-H/dMMR status, two are currently in clinical use: PCR to detect MSI-H and IHC to detect dMMR. These tests are widely used as a screening tool for the detection of Lynch syndrome and the evaluation of prognosis in patients with colorectal cancer. NGS of specific gene panels or whole exome sequencing is emerging as a potentially more analytically specific and sensitive method than PCR or IHC for determining MSI status [39,40]. Whereas traditional MSI testing relies on immunohistochemistry or fragment analysis of at least five microsatellites, 110 microsatellite markers are simultaneously evaluated by Action OncoKitDx with a 99\% correlation with the Bethesda panel.

The detection of MSI-H in the colorectal cancer patient could have diagnostic implications since it indicates suspicion of Lynch syndrome (LS), and is a prognostic factor of better survival outcome [41]. Furthermore, while different predictive biomarkers for immunotherapy have been investigated, including PD-L1, MSI/dMMR, and tumor mutational burden (TMB), MSI is currently the only pan-tumor biomarker for an approved oncological treatment. MSI-H in the colorectal tumor could thus provide access to the patient to the administration of Nivolumab or Pembrolizumab approved by the FDA or to clinical trials.

With respect to pharmacogenetics, $71.74 \%$ of the cases carried a clinically relevant SNP, up to current knowledge affecting response to different oncologic medications with a level of evidence of $2 \mathrm{~A}$ or superior, according to the PharmGKB The Pharmacogenomics Knowledgebase (PharmGKB).

\section{Discussion}

Action OncoKitDx, a hybrid capture NGS-based panel indicated in the study of adult solid tumors, was designed to fulfil the actual need of an integral analysis of all biomarkers related to available targeted treatments on the market, approved by international organizations such as the FDA or the EMA, or providing an alternative in recruiting clinical trials. The assay also studies genetic alterations with implications in prognosis, diagnosis, and resistance monitoring with robust and investigational evidence. For this purpose, somatic and germline alterations are analyzed across the complete sequence of 50 genes, 9 fusion genes, 110 microsatellite markers, and 10 pharmacogenetic SNPs.

In this study, we have validated analytical and clinical performance of the Action OncoKitDx in FFPE solid tumor samples, using a protocol that integrates the highly sensitive capture of regions of interest with hybridization probes, with the technique of molecular barcoding of each DNA fragment with a single adapter for high-performance massive sequencing (NGS). This type of protocol allows, during bioinformatic analysis, to carry out the efficient elimination of sequencing and PCR duplicates. The Action OncoKitDx demonstrated a 100\% sensitivity for all the biomarkers analyzed. Specificity was adequate, with an overall value of $98.4 \%$, being $95 \%$ for the MSI determination and greater than $99 \%$ for SNVs, structural variants, and pharmacogenetics. CNVs met the acceptance criterion of $100 \%$ concordance assessed by means of confirmation of variants detected with Action OncoKitDx through other methodologies (MLPA, dPCR, NGS panel). Results obtained highlight the robustness of the assay, with overall repeatability and reproducibility values greater than $99 \%$, being $99.15 \%$ and $98.3 \%$ respectively for SNV detection, while both exceeded $99.9 \%$ for CNVs, structural variants, MSI, and pharmacogenetics.

The assay enabled detection of variants in low-quality DNA samples as well as low allelic frequency mutations. In fact, the limit of detection set at $5 \%$ for SNVs, CNVs, and structural variants ensured detection in reference standards and clinical material in more than $90 \%$ of cases, thus meeting the acceptance criteria. For CNVs, the established LOD was 
3 copies for insertions and 1 copy for deletions, in samples with up to $50 \%$ infiltration with non-tumor cells. Similar assays designed for the detection of single-nucleotide variants, copy number alterations, and structural variants have been validated showing comparable results, thus supporting the analytical performance of the Action OncoKitDx [42].

The self-developed analytical platform DataGenomics provides an automatic report that includes functional and clinical classification of variants inspired by the Commission of Personalized Medicine (ComPerMed)'s two-level approach, as well as information of clinical relevance for the oncologist's decision-making provided by the mutational profile of the patient's tumor. The genetic report finally includes all the relevant information for tailoring personalized patient treatment, including therapies approved by the EMA or FDA agencies and available clinical trials.

Among the 166 samples selected from different hospitals, only $150(90 \%)$ were finally included in the clinical feasibility study cohorts. Low quality of genomic DNA extracted from FFPE tissue specimens is one of the main disadvantages of this form of preservation and preparation technique. In order to avoid the risk of introducing analytical errors, sequencings not meeting the acceptance criteria were excluded from the validation. A total of 141 different pathogenic variants were detected across the sample cohorts, corresponding to $97 \%$ of the cases. Despite the limited number of samples in our clinical feasibility cohorts, comparison with the TCGA data showed consistency with the tumor types with the largest number of specimens. The mutational landscape was coherent, even detecting the most frequent mutations at similar prevalences.

Comprehensive molecular profiling has demonstrated significant advantages over the standard practices as individual gene or hotspot testing, or small gene panels used for the recognition of the main mutations with approved therapies. The approach used by NGS-based panels such as the Action OncoKitDx, enable detection of less frequent actionable alterations, mutations with no associated treatment but influence on prognosis, resistance to therapy or diagnosis, or can even provide clinically relevant knowledge on pathogenic variants potentially becoming future therapeutic targets that could identify patients eligible for clinical trials. Among the 150 different pathogenic variants identified, 82 presented clinical significance in their respective tumor types according to the four-tiered ACMG/AMP guideline system, allowing guidance in treatment strategy in $82.7 \%$ of cases.

Extensive genomic profiling has proven better reliability in identifying colorectal patients with resistance-associated KRAS alterations, melanoma patients with responsive BRAF mutations, lung cancer patients with therapy-associated $A L K$ rearrangements, and ovarian cancer patients with somatic BRCA1/2 alterations who may benefit from PARP inhibitors, than the respective traditional approaches [43-46]. This clinical feasibility study consistently detected not only non-hotspot actionable alterations who may identify patients benefiting from associated therapies that would not be indicated otherwise, but also all deleterious mutations in the codifying region of the targeted genes.

Besides clinically relevant SNVs, the assay identified different actionable biomarkers, including large and focal CNVs, fusions, MSI-H tumors and, in more than half of the cases, pharmacogenetic genotype-phenotype associations related to response to oncological drugs. MSI-H determination regardless of the tumor type, potentially provides therapeutic options to cancer types where microsatellite instability status would not be routinely determined owing to low incidence. In the research for identification of the most appropriate predictive biomarkers for immunotherapy, TMB and MSI evaluation are emerging as more reliable methods than PDL-1 expression detection, currently used as indication criterion for administration of some checkpoint inhibitors [47-50]. MSI has become the first pancancer predictive biomarker for an FDA-approved therapy (pembrolizumab) and provides prognostic guidance in some tumor types.

While integrating the analysis of different genomic alterations in a single test, the Action OncoKitDx provided a cost-effective, sample and time-saving alternative to the requirement of distinct molecular methodologies, thereby highlighting the importance of the translation of comprehensive genomic profiling into clinical practice. 
Among the analytical limitations encountered, the technology used did not allow distinguishing between high-homology regions such as pseudogenes, leading to false positives or negatives. Moreover, below the established quality parameters, specificity, sensitivity, reproducibility, and repeatability of results cannot be guaranteed. NGS technology is not yet considered the "Gold Standard" technique for some types of alterations, so it is recommended, whenever possible, to confirm positive results using complementary and standardized technology. Since this approach has been developed for variants of somatic origin, under suspicion of a germline origin the validation in a non-tumoral sample of the patient (i.e., peripheral blood or normal tissue) is recommended. In case of a clinically interesting finding, specific genetic counselling should be performed due to its hereditary component. Recommendations related to drugs or clinical trials should be taken as a reference for its clinical evaluation and interpretation by the oncologist, in an integrated manner, together with the rest of the patient's clinical information and results from complementary analytical or imaging tests.

Taken together, our results confirmed compliance of the acceptance criteria established for each analytical parameter, thus validating the suitability of the Action OncoKitDx for the identification of somatic mutations in FFPE solid tumor samples. Herein we validate that the present assay allows the sensitive and specific simultaneous analysis of different actionable alterations and that the technical protocol and the bioinformatic pipeline of the Action OncoKitDx are repeatable and reproducible. Our experience from the implementation of the Action OncoKitDx in the clinical setting has shown great advantage over traditional routine genetic testing approaches. The assay efficiently reports the main driver events and generates valuable information for clinical decision-making.

Author Contributions: Conceptualization, P.M.-F., P.P., R.D.-G., A.G., I.T.-S., J.V.-G., C.R., M.L., P.C., P.G., C.M., J.J.R.-M., N.C.-L., J.F.-V., I.C.; methodology, J.V.-G., C.R., I.C.; software, J.V.-G., C.R.; validation, P.M.-F., M.G.-R., I.B., M.I.-G., J.V.-G., C.R., M.L., P.C., P.G., I.C.; formal analysis, P.M.-F., M.L., P.C., P.G., I.C.; investigation, M.G.-R., I.B., M.I.-G., P.P., R.D.-G., A.G., I.T.-S., E.R.-Z., C.M., J.J.R.-M., N.C.-L., J.F.-V.; resources, P.P., R.D.-G., A.G., I.T.-S., E.R.-Z., C.M., J.J.R.-M., A.P.-U., N.C.-L., J.F.-V.; data curation, J.V.-G., C.R.; writing-original draft preparation, P.M.-F.; writing-review and editing, all authors; visualization, P.M.-F., P.G., M.I.-G., J.V.-G., I.C.; supervision, I.C.; project administration, I.C.; funding acquisition, I.C. All authors have read and agreed to the published version of the manuscript.

Funding: This research received no external funding.

Institutional Review Board Statement: The study was conducted according to the guidelines of the Declaration of Helsinki, and approved by the Ethics Committee of Clinical Investigation of the University Hospita Peset (CEIC code 29/18 and date of approval 21 March 2018).

Informed Consent Statement: Informed consent was obtained from all subjects involved in the study.

Data Availability Statement: The data presented in this study are available within the article.

Acknowledgments: The authors would like to thank to Cristian Pérez, Angel Luis Campos and Javier García-Planells for their technical support and advise.

Conflicts of Interest: The authors Paula Martínez-Fernández, María José García, Ibon Barba, Marta Izquierdo-García, Jennifer Valero-Garcia, Carlos Ruiz, Marián Lázaro, Paula Carbonell, Pablo Gargallo, Inés Calabria are Imegen (Health In Code group) employees. The other authors declare no conflict of interest.

\section{References}

1. Lee, Y.T.; Tan, Y.J.; Oon, C.E. Molecular Targeted Therapy: Treating Cancer with Specificity. Eur. J. Pharmacol. 2018, 834, 188-196. [CrossRef] [PubMed]

2. Kamps, R.; Brandão, R.; Bosch, B.; Paulussen, A.; Xanthoulea, S.; Blok, M.; Romano, A. Next-Generation Sequencing in Oncology: Genetic Diagnosis, Risk Prediction and Cancer Classification. Int. J. Mol. Sci. 2017, 18, 308. [CrossRef] [PubMed]

3. Sobhani, N.; Roviello, G.; Corona, S.P.; Scaltriti, M.; Ianza, A.; Bortul, M.; Zanconati, F.; Generali, D. The Prognostic Value of PI3K Mutational Status in Breast Cancer: A Meta-analysis. J. Cell. Biochem. 2018, 119, 4287-4292. [CrossRef] [PubMed]

4. Markham, A. Alpelisib: First Global Approval. Drugs 2019, 79, 1249-1253. [CrossRef] 
5. André, F.; Ciruelos, E.; Rubovszky, G.; Campone, M.; Loibl, S.; Rugo, H.S.; Iwata, H.; Conte, P.; Mayer, I.A.; Kaufman, B.; et al. Alpelisib for PIK3CA-Mutated, Hormone Receptor-Positive Advanced Breast Cancer. N. Engl. J. Med. 2019, 380, $1929-1940$. [CrossRef]

6. Huang, D.; Tang, L.; Yang, F.; Jin, J.; Guan, X. PIK3CA Mutations Contribute to Fulvestrant Resistance in ER-Positive Breast. Am. J. Transl. Res. 2019, 11, 6055.

7. Rimawi, M.F.; De Angelis, C.; Contreras, A.; Pareja, F.; Geyer, F.C.; Burke, K.A.; Herrera, S.; Wang, T.; Mayer, I.A.; Forero, A.; et al Low PTEN Levels and PIK3CA Mutations Predict Resistance to Neoadjuvant Lapatinib and Trastuzumab without Chemotherapy in Patients with HER2 Over-Expressing Breast Cancer. Breast Cancer Res. Treat 2018, 167, 731-740. [CrossRef]

8. Kataoka, Y.; Mukohara, T.; Shimada, H.; Saijo, N.; Hirai, M.; Minami, H. Association between Gain-of-Function Mutations in PIK3CA and Resistance to HER2-Targeted Agents in HER2-Amplified Breast Cancer Cell Lines. Ann. Oncol. 2010, 21, $255-262$. [CrossRef]

9. Olivier, M.; Langer, A.; Carrieri, P.; Bergh, J.; Klaar, S.; Eyfjord, J.; Theillet, C.; Rodriguez, C.; Lidereau, R.; Bi, I.; et al. The Clinical Value of Somatic TP53 Gene Mutations in 1794 Patients with Breast Cancer. Clin. Cancer Res. 2006, 12, 1157-1167. [CrossRef] [PubMed]

10. Silwal-Pandit, L.; Vollan, H.K.M.; Chin, S.-F.; Rueda, O.M.; McKinney, S.; Osako, T.; Quigley, D.A.; Kristensen, V.N.; Aparicio, S.; Børresen-Dale, A.-L.; et al. TP53 Mutation Spectrum in Breast Cancer Is Subtype Specific and Has Distinct Prognostic Relevance. Clin. Cancer Res. 2014, 20, 3569-3580. [CrossRef]

11. Xie, M.; Li, J.; Cai, Z.; Li, K.; Hu, B. Impact of Primary Colorectal Cancer Location on the KRAS Status and Its Prognostic Value. BMC Gastroenterol. 2019, 19, 46. [CrossRef] [PubMed]

12. Yoon, H.H.; Tougeron, D.; Shi, Q.; Alberts, S.R.; Mahoney, M.R.; Nelson, G.D.; Nair, S.G.; Thibodeau, S.N.; Goldberg, R.M.; Sargent, D.J.; et al. KRAS Codon 12 and 13 Mutations in Relation to Disease-Free Survival in BRAF-Wild-Type Stage III Colon Cancers from an Adjuvant Chemotherapy Trial (N0147 Alliance). Clin. Cancer Res. 2014, 20, 3033-3043. [CrossRef] [PubMed]

13. Marchand, A.; Tallet, A.; Collin, C.; Cormier, B.; Venel, Y.; Miquelestorena-Standley, E.; Machet, L. A Rare BRAF T599dup Mutation Conferring Sensitivity to BRAF Inhibitor in a Patient with Metastatic Melanoma. Br. J. Dermatol. 2018, 179, 528-529. [CrossRef] [PubMed]

14. Mu, Y.; Yang, K.; Hao, X.; Wang, Y.; Wang, L.; Liu, Y.; Lin, L.; Li, J.; Xing, P. Clinical Characteristics and Treatment Outcomes of 65 Patients With BRAF-Mutated Non-Small Cell Lung Cancer. Front. Oncol. 2020, 10, 603. [CrossRef] [PubMed]

15. Carvajal, R.D.; Lawrence, D.P.; Weber, J.S.; Gajewski, T.F.; Gonzalez, R.; Lutzky, J.; O’Day, S.J.; Hamid, O.; Wolchok, J.D.; Chapman, P.B.; et al. Phase II Study of Nilotinib in Melanoma Harboring KIT Alterations Following Progression to Prior KIT Inhibition. Clin. Cancer Res. 2015, 21, 2289-2296. [CrossRef]

16. Balch, C.M.; Soong, S.; Gershenwald, J.E.; Thompson, J.F.; Coit, D.G.; Atkins, M.B.; Ding, S.; Cochran, A.J.; Eggermont, A.M.M.; Flaherty, K.T.; et al. Age as a Prognostic Factor in Patients with Localized Melanoma and Regional Metastases. Ann. Surg. Oncol. 2013, 20, 3961-3968. [CrossRef]

17. Maurichi, A.; Miceli, R.; Camerini, T.; Mariani, L.; Patuzzo, R.; Ruggeri, R.; Gallino, G.; Tolomio, E.; Tragni, G.; Valeri, B.; et al. Prediction of Survival in Patients With Thin Melanoma: Results From a Multi-Institution Study. J. Clin. Oncol. 2014, 32, $2479-2485$. [CrossRef]

18. Eriksson, H.; Frohm-Nilsson, M.; Järås, J.; Kanter-Lewensohn, L.; Kjellman, P.; Månsson-Brahme, E.; Vassilaki, I.; Hansson, J. Prognostic Factors in Localized Invasive Primary Cutaneous Malignant Melanoma: Results of a Large Population-Based Study. Br. J. Dermatol. 2015, 172, 175-186. [CrossRef]

19. Queirolo, P.; Spagnolo, F. Binimetinib for the Treatment of NRAS-Mutant Melanoma. Expert Rev. Anticancer Ther. 2017, 17, 985-990. [CrossRef]

20. Dummer, R.; Schadendorf, D.; Ascierto, P.A.; Arance, A.; Dutriaux, C.; Di Giacomo, A.M.; Rutkowski, P.; Del Vecchio, M.; Gutzmer, R.; Mandala, M.; et al. Binimetinib versus Dacarbazine in Patients with Advanced NRAS-Mutant Melanoma (NEMO): A Multicentre, Open-Label, Randomised, Phase 3 Trial. Lancet Oncol. 2017, 18, 435-445. [CrossRef]

21. Sarkisian, S.; Davar, D. MEK Inhibitors for the Treatment of NRAS Mutant Melanoma. Drug Des. Dev. Ther. 2018, 12, 2553-2565. [CrossRef] [PubMed]

22. Specenier, P. An Overview of Binimetinib for the Treatment of Melanoma. Expert Opin. Pharmacother. 2020, 21, 747-754. [CrossRef] [PubMed]

23. Buscail, L.; Bournet, B.; Cordelier, P. Role of Oncogenic KRAS in the Diagnosis, Prognosis and Treatment of Pancreatic Cancer. Nat. Rev. Gastroenterol. Hepatol. 2020, 17, 153-168. [CrossRef] [PubMed]

24. Wu, K.; Zhang, X.; Li, F.; Xiao, D.; Hou, Y.; Zhu, S.; Liu, D.; Ye, X.; Ye, M.; Yang, J.; et al. Frequent Alterations in Cytoskeleton Remodelling Genes in Primary and Metastatic Lung Adenocarcinomas. Nat. Commun. 2015, 6, 10131. [CrossRef] [PubMed]

25. Scoccianti, C.; Vesin, A.; Martel, G.; Olivier, M.; Brambilla, E.; Timsit, J.-F.; Tavecchio, L.; Brambilla, C.; Field, J.K.; Hainaut, P.; et al. Prognostic Value of TP53, KRAS and EGFR Mutations in Nonsmall Cell Lung Cancer: The EUELC Cohort. Eur. Respir. J. 2012, 40, 177-184. [CrossRef] [PubMed]

26. Lee, S.Y.; Jeon, H.-S.; Hwangbo, Y.; Jeong, J.Y.; Park, J.Y.; Lee, E.J.; Jin, G.; Shin, K.M.; Yoo, S.S.; Lee, J.; et al. The Influence of TP53 Mutations on the Prognosis of Patients with Early Stage Non-Small Cell Lung Cancer May Depend on the Intratumor Heterogeneity of the Mutations: Intratumor heterogeneity of tp53 mutations in NSCLC. Mol. Carcinog. 2015, 54, 93-101. [CrossRef] 
27. Santos, C.; Sanz-Pamplona, R.; Salazar, R. RET-Fusions: A Novel Paradigm in Colorectal Cancer. Ann. Oncol. 2018, 29 , 1340-1343. [CrossRef] [PubMed]

28. Perez-Garcia, J.; Muñoz-Couselo, E.; Soberino, J.; Racca, F.; Cortes, J. Targeting FGFR Pathway in Breast Cancer. Breast 2018, 37, 126-133. [CrossRef] [PubMed]

29. Helsten, T.; Elkin, S.; Arthur, E.; Tomson, B.N.; Carter, J.; Kurzrock, R. The FGFR Landscape in Cancer: Analysis of 4853 Tumors by Next-Generation Sequencing. Clin. Cancer Res. 2016, 22, 259-267. [CrossRef]

30. Elbauomy Elsheikh, S.; Green, A.R.; Lambros, M.B.; Turner, N.C.; Grainge, M.J.; Powe, D.; Ellis, I.O.; Reis-Filho, J.S. FGFR1 Amplification in Breast Carcinomas: A Chromogenic in Situhybridisation Analysis. Breast Cancer Res. 2007, 9, R23. [CrossRef]

31. Kraehn, G.M.; Utikal, J.; Udart, M.; Greulich, K.M.; Bezold, G.; Kaskel, P.; Leiter, U.; Peter, R.U. Extra C-Myc Oncogene Copies in High Risk Cutaneous Malignant Melanoma and Melanoma Metastases. Br. J. Cancer 2001, 84, 72-79. [CrossRef]

32. Levva, S.; Kotoula, V.; Kostopoulos, I.; Manousou, K.; Papadimitriou, C.; Papadopoulou, K.; Lakis, S.; Koukoulias, K.; Karavasilis, V.; Pentheroudakis, G.; et al. Prognostic Evaluation of Epidermal Growth Factor Receptor (EGFR) Genotype and Phenotype Parameters in Triple-Negative Breast Cancers. Cancer Genom. Proteom. 2017, 14, 181-195. [CrossRef] [PubMed]

33. Gossage, L.; Eisen, T.; Maher, E.R. VHL, the Story of a Tumour Suppressor Gene. Nat. Rev. Cancer 2015, 15, 55-64. [CrossRef] [PubMed]

34. Kroeger, P.T.; Drapkin, R. Pathogenesis and Heterogeneity of Ovarian Cancer. Curr. Opin. Obstet. Gynecol. 2017, $29,26-34$. [CrossRef] [PubMed]

35. Etemadmoghadam, D.; Weir, B.A.; Au-Yeung, G.; Alsop, K.; Mitchell, G.; George, J.; Australian Ovarian Cancer Study Group; Davis, S.; D'Andrea, A.D.; Simpson, K.; et al. Synthetic Lethality between CCNE1 Amplification and Loss of BRCA1. Proc. Natl. Acad. Sci. USA 2013, 110, 19489-19494. [CrossRef] [PubMed]

36. De' Angelis, G.L.; Bottarelli, L.; Azzoni, C.; de' Angelis, N.; Leandro, G.; Di Mario, F.; Gaiani, F.; Negri, F. Microsatellite Instability in Colorectal Cancer. Acta Biomed. 2018, 89, 97-101. [CrossRef]

37. Kubeček, O.; Kopecký, J. Microsatellite Instability in Melanoma: A Comprehensive Review. Melanoma Res. 2016, 26, 545-550. [CrossRef] [PubMed]

38. Baretti, M.; Le, D.T. DNA Mismatch Repair in Cancer. Pharmacol. Ther. 2018, 189, 45-62. [CrossRef]

39. Stadler, Z.K.; Battaglin, F.; Middha, S.; Hechtman, J.F.; Tran, C.; Cercek, A.; Yaeger, R.; Segal, N.H.; Varghese, A.M.; Reidy-Lagunes, D.L.; et al. Reliable Detection of Mismatch Repair Deficiency in Colorectal Cancers Using Mutational Load in Next-Generation Sequencing Panels. J. Clin. Oncol. 2016, 34, 2141-2147. [CrossRef]

40. Waalkes, A.; Smith, N.; Penewit, K.; Hempelmann, J.; Konnick, E.Q.; Hause, R.J.; Pritchard, C.C.; Salipante, S.J. Accurate Pan-Cancer Molecular Diagnosis of Microsatellite Instability by Single-Molecule Molecular Inversion Probe Capture and HighThroughput Sequencing. Clin. Chem. 2018, 64, 950-958. [CrossRef]

41. Mármol, I.; Sánchez-de-Diego, C.; Pradilla Dieste, A.; Cerrada, E.; Rodriguez Yoldi, M. Colorectal Carcinoma: A General Overview and Future Perspectives in Colorectal Cancer. Int. J. Mol. Sci. 2017, 18, 197. [CrossRef] [PubMed]

42. Garcia, E.P.; Minkovsky, A.; Jia, Y.; Ducar, M.D.; Shivdasani, P.; Gong, X.; Ligon, A.H.; Sholl, L.M.; Kuo, F.C.; MacConaill, L.E.; et al. Validation of OncoPanel: A Targeted Next-Generation Sequencing Assay for the Detection of Somatic Variants in Cancer. Arch. Pathol. Lab. Med. 2017, 141, 751-758. [CrossRef] [PubMed]

43. Rankin, A.; Klempner, S.J.; Erlich, R.; Sun, J.X.; Grothey, A.; Fakih, M.; George, T.J.; Lee, J.; Ross, J.S.; Stephens, P.J.; et al. Broad Detection of Alterations Predicted to Confer Lack of Benefit from EGFR Antibodies or Sensitivity to Targeted Therapy in Advanced Colorectal Cancer. Oncologist 2016, 21, 1306-1314. [CrossRef]

44. Boussemart, L.; Nelson, A.; Wong, M.; Ross, J.S.; Sosman, J.; Mehnert, J.; Daniels, G.; Kendra, K.; Ali, S.M.; Miller, V.A.; et al. Hybrid Capture-Based Genomic Profiling Identifies BRAF V600 and Non-V600 Alterations in Melanoma Samples Negative by Prior Testing. Oncologist 2019, 24, 657-663. [CrossRef] [PubMed]

45. Drilon, A.; Wang, L.; Arcila, M.E.; Balasubramanian, S.; Greenbowe, J.R.; Ross, J.S.; Stephens, P.; Lipson, D.; Miller, V.A.; Kris, M.G.; et al. Broad, Hybrid Capture-Based Next-Generation Sequencing Identifies Actionable Genomic Alterations in Lung Adenocarcinomas Otherwise Negative for Such Alterations by Other Genomic Testing Approaches. Clin. Cancer Res. 2015, 21, 3631-3639. [CrossRef]

46. Ali, S.M.; Hensing, T.; Schrock, A.B.; Allen, J.; Sanford, E.; Gowen, K.; Kulkarni, A.; He, J.; Suh, J.H.; Lipson, D.; et al. Comprehensive Genomic Profiling Identifies a Subset of Crizotinib-Responsive ALK-Rearranged Non-Small Cell Lung Cancer Not Detected by Fluorescence In Situ Hybridization. Oncologist 2016, 21, 762-770. [CrossRef]

47. Le, D.T.; Uram, J.N.; Wang, H.; Bartlett, B.R.; Kemberling, H.; Eyring, A.D.; Skora, A.D.; Luber, B.S.; Azad, N.S.; Laheru, D.; et al. PD-1 Blockade in Tumors with Mismatch-Repair Deficiency. N. Engl. J. Med. 2015, 372, 2509-2520. [CrossRef]

48. Masucci, G.V.; Cesano, A.; Hawtin, R.; Janetzki, S.; Zhang, J.; Kirsch, I.; Dobbin, K.K.; Alvarez, J.; Robbins, P.B.; Selvan, S.R.; et al. Validation of Biomarkers to Predict Response to Immunotherapy in Cancer: Volume I-Pre-Analytical and Analytical Validation. J. Immunother. Cancer 2016, 4, 76. [CrossRef]

49. Grigg, C.; Rizvi, N.A. PD-L1 Biomarker Testing for Non-Small Cell Lung Cancer: Truth or Fiction? J. Immunother. Cancer 2016, 4, 48. [CrossRef]

50. Madore, J.; Strbenac, D.; Vilain, R.; Menzies, A.M.; Yang, J.Y.H.; Thompson, J.F.; Long, G.V.; Mann, G.J.; Scolyer, R.A.; Wilmott, J.S. PD-L1 Negative Status Is Associated with Lower Mutation Burden, Differential Expression of Immune-Related Genes, and Worse Survival in Stage III Melanoma. Clin. Cancer Res. 2016, 22, 3915-3923. [CrossRef] 